

Kenia Rodríguez de Benítez*

\section{Implementación del proceso de producción escrita para el mejoramiento de la redacción de textos expositivos en Administración Hotelera}

\author{
Implementing the writing process to improve \\ Hotel Management students' writing of \\ expository texts
}

\title{
Resumen
}

El presente estudio de investigación-acción está enfocado en la implementación de la escritura como proceso para el mejoramiento de la redacción de textos expositivos con la estructura problema-solución, al haberse identificado la necesidad de ayudar a los estudiantes con sus reflexiones y lograr escribir textos con mayor coherencia, criticidad y análisis. La estrategia es aplicada en la asignatura Turismo Sostenible, en el período académico 1-2014-2015. Se utiliza la corrección procesal para las retroalimentaciones de los estudiantes y una rúbrica para evaluar. En los resultados se presenta una mejora significativa en los textos finales redactados por los estudiantes y la mitad de estos está de acuerdo en que el uso de esta estrategia los convirtió en mejores escritores.

\section{Abstract}

This article presents an action research study focused on the implementation of the writing process to improve students' writing of expository texts within the problem-solution essay structure. The general aim of the study was to help students write with greater consistency, criticality, and depth of analysis. The writing as a process strategy was implemented in the course Sustainable Tourism in the academic term 1-2014-2015. A rubric to assess students' writing was used and constant feedback was offered. Results show a significant improvement in the final written texts. Some students in this course agreed that the use of this strategy helped them become better writers.

\section{Palabras clave}

proceso de escritura; corrección procesal; textos expositivos

\section{Keywords}

writing process; feedback process; expository texts

\footnotetext{
*Kenia Rodríguez de Benítez: Licenciada en Administración Hotelera de la Pontificia Universidad Católica Madre y Maestra (PUCMM). Maestría en Gestión Turística con especialidad en Desarrollo Turístico Sostenible de la Universidad de las Islas Baleares, España. Profesora a tiempo completo y coordinadora académica docente del Departamento de Administración Hotelera del campus Santiago, PUCMM. Para contactar a la autora: knrodriguez@pucmm.edu.do
} 


\section{Introducción}

El presente artículo muestra los resultados del proyecto de investigación acción realizado en el marco del Diplomado en Lectura y Escritura a través del Currículum en el Nivel Superior, organizado por el Centro de Excelencia para la Investigación y Difusión de la Lectura y Escritura (CEDILE).

En este diplomado se trabajó profundamente el concepto de alfabetización académica, el cual es definido por Carlino (2013, p. 370) como "el proceso de enseñanza que puede (o no) ponerse en marcha para favorecer el acceso de los estudiantes a las diferentes culturas escritas de las disciplinas".

Este término está relacionado directamente con el desarrollo de las habilidades necesarias para lograr la reflexión dentro del ámbito universitario. Para conseguir estas habilidades son requeridas una serie de acciones, implementadas por los docentes en las aulas, que conlleven a formar estudiantes con altos niveles de criticidad. La estrategia de la escritura como proceso es una herramienta significativa y de alto impacto en el estudiantado, contribuyendo a facilitarles su espacio en la comunidad discursiva de la universidad.

La escritura representa grandes dificultades para los estudiantes, como lo expresa Huerta (2012, pp. 117118), "se encuentra la carencia de los estudiantes de educación superior, les falta una metodología o trabajo sistemático para abordar las actividades de escritura. Prueba de tal carencia son los innumerables proyectos de alfabetización o literacidad académica que algunas instituciones están implementado".

En este sentido, es el rol del docente guiar en el proceso y facilitar los instrumentos claves para obtener mejores resultados y aprendizajes más significativos.

A partir de esta premisa, se desarrolló el presente proyecto de investigación acción, cuyo propósito general fue mejorar la producción de textos expositivos con la estructura problema-solución mediante la estrategia de la escritura como proceso según los autores Flower y Hayes (1981) en los estudiantes de Administración Hotelera.

Los objetivos específicos de la investigación incluyeron: implementar el proceso de producción escrita en sus cuatro etapas (planificación, textualización, examen y control) según el modelo de expresión escrita de Flower y Hayes (1981) para la redacción de textos expositivos con la estructura problema-solución, así como aplicar rúbricas para las evaluaciones.

\section{Contextualización}

Los egresados de la carrera de Administración Hotelera deben desempeñarse dentro de un ambiente con grandes exigencias y expectativas por parte de los turistas, lo que implica contar con profesionales que sean capaces de responder efectivamente a esas demandas, a través de un plan curricular diseñado para lograr tales fines. Sin embargo, ya que su contexto es mayormente práctico y operacional, los elementos teóricos sobre el campo de trabajo son poco valorados por los estudiantes, presentando debilidades al momento de elaborar ideas, analizar información y reflexionar para la propuesta de soluciones y mejoras significativas.

Dentro de este contexto, se encuentran los estudiantes de la asignatura Turismo Sostenible (ADH-335), la cual es electiva y se ofrece al menos en dos períodos académicos durante el año. Según el programa académico de la asignatura, contenido en el Plan de Estudios de la Carrera Administración Hotelera, el objetivo general de ésta es "aprender cómo el ecoturismo puede ser usado como una estrategia sostenible para generar positivamente impactos ambientales, sociales y económicos sobre las comunidades, la biodiversidad y las áreas protegidas o naturales" Plan de Estudios de la Carrera Administración Hotelera (2012).

La base teórica usada en la asignatura es un Manual para el Ecoturismo dirigido a profesionales del mismo, elaborado por Nature Conservancy. Este manual contiene una gran cantidad de casos de estudio, a partir de los cuales los estudiantes deben realizar análisis y proponer recomendaciones.

Durante el desarrollo de la asignatura, se visita un proyecto ecoturístico del país donde los estudiantes pueden ver, aplicadas en la realidad, las estrategias de turismo sostenible que involucran a las comunidades locales. Sobre esta visita deben redactar un reporte donde reflexionan sobre la teoría vista en clase, con lo observado durante la misma.

Como proyecto final los estudiantes deben elegir un área protegida del país y proponer un Plan de Manejo del Ecoturismo en el cual apliquen estrategias de turismo sostenible que den respuesta las problemáticas presentes en esa zona.

Tanto en la resolución de casos en clase, como en los proyectos finales asignados, se evidenció la debilidad de los estudiantes para redactar coherentemente y proponer soluciones viables, razonadas y bien pensadas. Los estudiantes se enfocan sólo en la teoría de la 
clase, no logran elaborar recomendaciones apropiadas según el contexto y responden con información explícita, sin análisis, ni reflexión.

Tomando en cuenta la situación descrita anteriormente, se identificó la necesidad de ayudar a los estudiantes a mejorar sus reflexiones y lograr escribir textos con mayor coherencia, criticidad y análisis.

\section{Antecedentes del estudio}

El área de estudio de esta investigación es la escritura como proceso. A nivel local e internacional existen algunas publicaciones sobre estudios relacionados con este tema. Se seleccionaron tres estudios para ser comentados por considerarlos los más relacionados con el presente proyecto de investigación-acción.

Jesús Caos Huerta Rodríguez, partiendo de la escritura como proceso según el modelo de Flower y Hayes (1981), aplicó estrategias en un grupo de 22 estudiantes de Educación Superior para ayudarles en la planeación de ensayos en un período académico. El estudio reveló que "escribir por proceso no es suficiente para hacerlos buenos escritores" y que "las prácticas de escritura exigidas por la escuela no están relacionadas con las actividades de escritura de los estudiantes". (Huerta, 2012).

Otro estudio, a nivel de estudiantes de doctorado, fue el realizado por Castelló et al (2009), denominado "Hacia la escritura autorregulada: un estudio exploratorio con estudiantes posgraduados en un entorno de aprendizaje situado". El objetivo del estudio fue "conocer las dificultades y estrategias efectivas que 19 estudiantes de doctorado utilizan a la hora de regular el proceso de escritura de sus textos académicos". Se hizo un análisis de sus concepciones sobre la escritura académica, estrategias de revisión en los borradores de los textos y la calidad de la versión final de los mismos. Los resultados revelaron que "la regulación y la calidad de los textos están relacionadas con la capacidad de los escritores para relacionar sus preocupaciones con soluciones y estrategias explícitas". (Castelló et al, 2009).

Dentro del contexto de la Pontificia Universidad Católica Madre y Maestra (PUCMM), se tomó como referencia la investigación realizada por la profesora Sonja Arias, en el marco de la primera cohorte del Diplomado en Lectura y Escritura a través del Currículo en el Nivel Superior del año 2013, cuyo objetivo general era "mejorar la producción escrita de informes de Pasantía Empresarial mediante la estrategia de la escritura como proceso" Arias (2013, p. 4). La profesora Arias utilizó la explicitación de guías, preguntas de verificación, corrección procesal, corrección por pares y evaluación mediante rúbricas. Algunas de sus conclusiones fueron que "los resultados logrados mediante la implementación de las estrategias innovadoras de escritura son satisfactorios" y que "algunos de los estudiantes que participaron en el proyecto consideran que se convirtieron en mejores escritores" Arias (2013, p. 16).

\section{Referentes teóricos}

Al pensar en la definición de la palabra "escritor", la mente humana normalmente recurre a aquel concepto enfocado en la capacidad de escribir libros de algún género literario como novelas. Sin embargo, este concepto va más allá, abarcando la habilidad de redactar efectivamente cualquier tipo de texto. Como expresan Cassany, Luna y Sanz (2002, p. 257), “... es un buen redactor o escritor quien es capaz de comunicarse coherentemente por escrito, produciendo textos de una extensión considerable sobre un tema de cultura general".

Tomando este concepto como referencia, se puede decir que, los estudiantes, en sentido general, deberían ser capaces de producir textos según los géneros discursivos en su área disciplinar de la forma más coherente posible. Los docentes suelen esperar un alto nivel de rendimiento en relación a este tema, teniendo altas expectativas sobre las producciones escritas de los estudiantes. Sin embargo, este proceso no es tan sencillo como parece, ya que, para poder escribir, es necesario contar con una serie de microhabilidades, agrupadas por Cassany, Luna y Sanz (2002) en conceptos (o saberes), procedimientos (o saber hacer) y actitudes.

Debido a la importancia de estas habilidades, este proyecto se enfoca, dentro de los procedimientos, en los aspectos cognitivos como la planificación, generación de ideas, formulación de objetivos, redacción y revisión. Sobre el proceso cognitivo de la escritura, Flower y Hayes (1981) desarrollan una teoría en la que se rompe el paradigma tradicional de la escritura y se evoluciona a un modelo que involucra procesos mentales básicos como por ejemplo, la generación de ideas. En este trabajo se tratan también, de manera más general, elementos del concepto del texto como la coherencia, cohesión y gramática.

En este mismo sentido, se espera que, a través de la escritura, los estudiantes sean capaces de reflexionar, de generar ideas adaptadas a su realidad y al contexto en general. Cassany (1999, p. 16), considera que "escribir es un poderoso instrumento de reflexión y que en el acto de escribir, los redactores aprenden sobre sí mismos, sobre su mundo y comunican sus 
percepciones a otros". Entonces, es válido preguntarse qué papel juegan los docentes en este proceso y cuáles serían sus principales funciones y responsabilidades para lograr la reflexión y el análisis crítico en los estudiantes.

Antes de abordar el rol desempeñado por el docente, es importante conocer en qué consiste el proceso de escritura más a fondo. Como citan Padilla, Douglas y López (2010), a pesar de que autores como Scardamalia y C. Bereiter $(1987,1992)$ han realizado aportes en relación a este tema determinando las diferencias en el proceso de composición de escritores novatos y expertos, "el modelo teórico más difundido y aplicado a la enseñanza es el de Flower y Hayes" (Cassany, Luna \& Sanz, 2002, p. 263).

El modelo de Flower y Hayes es bastante completo, explicativo, detallado $y$, además, "parte de la existencia de tres grandes unidades en el proceso global de producción escrita que interactúan y se retroalimentan constantemente de manera recursiva". (Padilla, Douglas \& López, 2010, p. 57). Las unidades del proceso son: el ambiente de trabajo, la memoria a largo plazo del escritor y los procesos de escritura propiamente dichos. Esta combinación de elementos no puede darse por separado. Estos aspectos deben integrarse mutuamente para poco a poco convertirse en un producto final con la mejor calidad posible. Además, hay que tomar en cuenta que este proceso no necesariamente es estático y lineal, sino que puede invertirse según la necesidad del escritor.

Otro concepto manejado en este trabajo, son los textos expositivos. Los textos expositivos, circulan en el ámbito educativo, su propósito es la comprensión y aprendizaje de aspectos disciplinarios del saber científico, teniendo como destinatarios estudiantes de todos los niveles y público en general. Siendo éstos los más comunes en el área de estudio de este proyecto, la estructura o modo de organización del discurso siempre trae muchas interrogantes para los estudiantes y es necesario orientarlos más a fondo sobre este tema. Meyer (1985), citada por Padilla, Douglas y López (2010), no postula una única estructura posible, sino cinco modos de organización expositiva que describe siguiendo el orden de un menor a un mayor grado de elaboración informativa: descripción, seriación, organización causal, problema/solución y comparación.

Como explica B. Meyer (1985), citada por Padilla, Douglas y López (2010, p. 24), dentro del modo de organización problema/solución "un vínculo causal puede ser parte del problema o de la solución. Los textos pueden tener indicadores de esta organización, tales como: un problema que debe resolverse es..., las soluciones que se proponen... La autora señala que esta forma de organización es habitual en textos sobre asuntos humanos: ciencias sociales o biología".

Agregando a esto, dentro de esta estructura, "los problemas se plantean y se explican a través de relaciones de causalidad; es decir, los problemas se constituyen en causas que desencadenan un determinado efecto, el que a su vez provoca otro efecto" (Padilla, Douglas y López, 2010, p. 26).

A partir de esta descripción, se puede notar que la estructura problema/solución requiere un mayor nivel de elaboración de información y de reflexión, siendo apta para este proyecto, ya que colabora con el objetivo de lograr un aprendizaje más significativo, a través del análisis profundo y detallado para presentar soluciones, estableciendo relaciones de causalidad, haciendo propuestas de mejora en los estudios de caso y proyectos que se presentan en la asignatura en cuestión.

La gran variedad y diferencia de los escritos de los estudiantes enriquece grandemente el proceso de enseñanza-aprendizaje, ya que, a pesar de contar con los mismos problemas dentro de un texto, las posibles soluciones pueden presentar gran diversidad, aportando nuevas perspectivas sobre un tema en particular. Está claro que "cada alumno tiene que desarrollar su propio estilo y su método de trabajo, de acuerdo con su carácter y sus capacidades personales" (Cassany, Luna \& Sanz, 2002, p. 261). Es un compromiso del docente motivar y guiar al estudiante en este trayecto.

Por este motivo, aplicar el proceso de la escritura con la estructura problema-solución representa una oportunidad para la generación de ideas innovadoras y el aprendizaje significativo. A fin de cuentas, este es uno de los principales objetivos que todo docente debe tener dentro de la enseñanza-aprendizaje.

\section{Metodología}

Para la realización de este estudio se seleccionó la muestra arbitrariamente, eligiendo un grupo control y un grupo experimental.

El grupo experimental estaba compuesto por los estudiantes de la asignatura ADH-335-T-002 Turismo Sostenible, en el período académico 1-2014-2015, con un total de 20 estudiantes, 13 mujeres y 7 hombres. En el grupo había 4 estudiantes cuyo idioma natal no es el español. El $80 \%$ de los estudiantes estaba cursando el último año de la carrera. Del total de los estudiantes, sólo se tomaron en cuenta aquellos que cumplieron con el tiempo de entrega del borrador para ser retroalimentados. También, se descartaron algunos 
trabajos que fueron plagiados entre estudiantes o entregados incompletos. El número final de estudiantes que aplicaron la estrategia fue de 11.

Se seleccionó como grupo control ADH-335-T-001 Turismo Sostenible en el período académico 1-20142015. Estaba compuesto por 19 estudiantes, 16 mujeres y 3 hombres. En el grupo había 1 estudiante cuyo idioma natal no es el español. El 50\% de los estudiantes estaban cursando el tercer y cuarto año de la carrera.

La estrategia seleccionada para el estudio fue el proceso de producción escrita para la redacción de textos expositivos con la estructura problema-solución. Se aplicó de manera individual, en dos fases: borrador y producto final, durante las semanas 9 y 14 de clases. Se elaboró un documento guía con instrucciones específicas para la resolución de un caso de estudio y redacción de un texto con la estructura problemasolución, siguiendo los pasos del proceso de producción escrita.

Estos pasos son descritos por Cassany, Luna y Sanz (2002, p. 285), citando a Flower (1989): contextualización, gestación de ideas, planificación del texto, redacción del borrador y producto neto. En el primer paso, contextualización, el alumno debe interrogarse sobre el sentido del escrito que quiere producir. ¿Qué se sabe del tema? ¿Cómo es el lector? ¿Cómo quiere presentarse el autor? En el segundo paso, generar ideas, se deben buscar datos, informaciones, fuentes de consulta, ideas y observaciones. El tercer paso es la planificación del texto, organizar las ideas haciendo un esquema de lo que se va a redactar. El cuarto paso se trata de la redacción del borrador utilizando el esquema creado, asegurándose de que se está cumpliendo con el objetivo planteado, dirigido al público correcto y asumiendo el rol definido como escritor. Finalmente, se debe crear el producto neto revisando el borrador y valorando lo que se ha escrito, releyendo críticamente.

La tabla 1 muestra un extracto de la guía entregada a los estudiantes, cuya versión completa incluye los pasos para el proceso de producción escrita, una descripción del caso a solucionar, un artículo de periódico como información extra sobre el caso, un ejemplo de un texto corto con la estructura problema-solución y la rúbrica de evaluación de textos expositivos.

La práctica se inició en el aula y luego los estudiantes redactaron los borradores en sus hogares. En el grupo control se solicitó a los estudiantes redactar el mismo texto, sin seguir los pasos del proceso de escritura, ni con el requerimiento de un borrador.

\section{Tabla 1}

Guía para el proceso de producción escrita entregada a los estudiantes

\section{PONTIFICIA UNIVERSIDAD CATÓLICA MADRE Y MAESTRA \\ FACULTAD DE CIENCIAS SOCIALES Y ADMINISTRATIVAS \\ DEPARTAMENTO DE ADMINISTRACIÓN HOTELERA \\ ADH-335-T-001 y 002 Turismo Sostenible}

\section{Caso \#6: Declaración del Archipiélago de Cabrera como Parque Nacional Marítimo Terrestre} Contextualización:

Usted es un consultor que ha sido contratado por el futuro equipo gestor del parque para analizar y presentar posibles soluciones al siguiente caso.

Este lugar aún no ha sido formalmente declarado como parque nacional. Usted debe ayudar a que este proceso se haga de la mejor manera posible para todos los actores involucrados.

Deberá presentar un documento de 1-2 páginas resumiendo la problemática y presentado las mejores soluciones.

Instrucciones generales

1. Lea todas las instrucciones y el recuadro antes de leer el caso para tener una idea de lo que debe contestar.

2. Antes de escribir, siga los pasos que le ayudarán a la redacción del texto a través del siguiente recuadro que orientará sus respuestas. 
(Continuación tabla 1)

\begin{tabular}{|l|l|}
\hline \multicolumn{2}{|c|}{ GUÍA PARA REDACTAR TEXTO PROBLEMA-SOLUCIÓN } \\
\hline PASO NO.1: CONTEXTUALIZACIÓN \\
\hline Definir objetivo & $\begin{array}{l}\text { Proponer soluciones a las problemáticas presentadas en } \\
\text { la declaración del Archipiélago de Cabrera como Parque } \\
\text { Nacional Marítimo Terrestre. }\end{array}$ \\
\hline Definir tema o idea central & \\
\hline Definir enunciador (quien escribe el texto) & \\
\hline Definir enunciatario (para quien escribe el texto) & \\
\hline
\end{tabular}

buscar datos, informaciones, fuentes de consulta, ideas, observaciones.

Lea los datos que se anexan sobre las características particulares del Archipiélago de Cabrera.

Busque las definiciones de las terminologías desconocidas por usted: actores, valores, uso público, comarca, tipos de zonificación, capacidad de carga, chárter, spots, pesca no reglada,etc.

Observe el ejemplo anexo de un texto parecido al que se le está pidiendo con la estructura problema-solución.

Identifique dentro del caso los siguientes puntos:

- Actores (stakeholders)

- Valores del parque afectados por el uso público

- Conflictos potenciales entre el parque y los actores

- Conflictos potenciales entre los actores

PASO NO.3: PLANIFICACIÓN DEL TEXTO

Haga un esquema del texto que va a redactar, incluyendo introducción, desarrollo y conclusión, recordando contestar los siguientes puntos:

- Estrategia de discusión que se pudieran usar entre el equipo gestor y los agentes locales.

- Posibles soluciones a cada uno de los conflictos (realice una lluvia de ideas).

- ¿Cuál será la estrategia de comunicación del plan a la comunidad?

PASO NO.4 REDACCIÓN DEL BORRADOR

Redactar el borrador del texto tomando como guía el esquema realizado anteriormente, asegurándose de que está cumpliendo con el objetivo planteado, dirigido al público correcto y asumiendo su rol de consultor.

Relea, revise y arregle el documento cuantas veces considere.

Utilice la rúbrica dada por la profesora para evaluar la calidad de su producción escrita.

\section{PASO NO.5 PRODUCTO NETO}

Evaluar el producto escrito. Prestar atención a la coherencia entre lo textualizado, los objetivos y los posibles destinatarios. No considerarlo como documento final hasta que no esté conforme $100 \%$. Utilice la rúbrica dada por la profesora para evaluar la calidad de su producción escrita. 
Continuación tabla 1. Guía para el proceso de producción escrita entregada a los estudiantes

\section{Descripción Caso \#6 \\ Declaración del Archipiélago de Cabrera como Parque Nacional Marítimo Terrestre}

El Archipiélago de Cabrera, ubicado en España, será declarado como Parque Nacional próximamente. Los siguientes datos describen su realidad actualmente:

Parque nacional marítimo terrestre, $85 \%$ de medio marino. Valores naturales tanto terrestres como marinos importantes. Zonas de reserva integral (no take-no entry zones). No existe más zonificación. Valor patrimonial cultural relevante. Valor afectivo-emocional. Elemento singular en la comarca.

Recibe unos 80.000 visitantes al año -datos no precisos-, 50\% en ferries; resto en embarcaciones particulares o chárter. No se conoce procedencia ni nacionalidad. No se ha elaborado un estudio de capacidad de carga. turismo.

Un único municipio. Entorno socioeconómico con nivel de renta alto, basado fundamentalmente en el

Empresas y particulares que interactúan económicamente con el parque antes de su declaración:

- Chárter de cruceros;

- Decenas de empresas de publicidad, que desean rodar spots;

- Dos empresas de ferries una desde un puerto de la vecindad (15 km del parque), la otra a $60 \mathrm{~km}$;

- Empresas de buceo: tres empresas, todas operando desde $60 \mathrm{~km}$ de distancia

- Pescadores: 61 embarcaciones de pesca profesional artesanal, procedentes de cuatro puertos base vecinos. No se practica la pesca turística reglada, aunque sí de forma irregular y no declarada.

Evalúe los datos descritos anteriormente y redacte un documento en el cual proponga las mejores soluciones a la problemática.

Caso presentado por el profesor José Amengual, Técnico del Parque Nacional del Archipiélago de Cabrera.

Para la revisión y evaluación de los textos se aplicó al grupo control y al grupo experimental la misma rúbrica que contenía los siguientes criterios: problemática (definir los problemas o dificultades del caso a solucionar y plantearla tomando en cuenta el contexto del mismo), objetivo (definir el propósito a lograr en el texto y evidenciarlo claramente a través del mismo), enunciador (tener claro el rol de quién escribe el texto) y destinatario (tomar en cuenta el enunciatario, es decir, a quién va dirigido el texto), superestructura global (poseer una estructura canónica que incluye introducción, desarrollo y conclusión de manera articulada), cohesión (la manera de relacionar y unir las distintas palabras, oraciones y párrafos del texto), coherencia y claridad (mantener el sentido global de la macroestructura), apego a la normativa (respeto a la acentuación, uso de letras, puntuación y concordancia) y soluciones propuestas (responder a la problemática mostrando el pensamiento crítico, reflexivo y analítico).

En la tabla 2 se puede ver el ejemplo de la rúbrica utilizada.

Los estudiantes entregaron los borradores, los cuales fueron revisados y comentados para su mejora, usando como referencia las recomendaciones de Cassany (2002) sobre la corrección procesal, quien detalla las diferencias entre la corrección tradicional y procesal, aclarando que esta última hace énfasis en el proceso, en el escritor, en el contenido y la forma, destacando el papel del maestro como colaborador con el alumno y finalmente, viendo la corrección como revisión y mejora de los textos.

Los estudiantes fueron instruidos para mejorar los borradores dentro del lapso de tiempo de una semana y entregar un producto final a partir de la retroalimentación realizada.

Para el análisis de los resultados se utilizaron las siguientes herramientas de medición:

- Comparación de las evaluaciones obtenidas por los estudiantes del grupo experimental en el borrador y en el producto final, usando la rúbrica de evaluación.

- Comparación de las evaluaciones obtenidas en el caso asignado entre el grupo de control y el grupo experimental, usando la rúbrica de evaluación.

- Cuestionario aplicado a los estudiantes diseñado en base al objetivo de conocer su apreciación sobre la estrategia de la escritura como proceso (ver tabla $3)$. 


\section{PONTIFICIA UNIVERSIDAD CATÓLICA MADRE Y MAESTRA \\ FACULTAD DE CIENCIAS SOCIALES Y ADMINISTRATIVAS DEPARTAMENTO DE ADMINISTRACIÓN HOTELERA ADH-335-T-001 y 002 Turismo Sostenible}

RÚBRICA PARA EVALUACIÓN DE UN TEXTO EXPOSITIVO PROBLEMA-SOLUCIÓN: Adaptada a la usada por la prof. Montenegro.

\begin{tabular}{|c|c|c|c|}
\hline EVALUACIÓN & MAYORMENTE LOGRADO & MEDIANAMENTE LOGRADO & NO LOGRADO \\
\hline $\begin{array}{l}\text { Problemática } \\
\text { (1) }\end{array}$ & $\begin{array}{l}\text { La problemática está bien } \\
\text { planteada, resumida de manera } \\
\text { clara y coherente, y tomando en } \\
\text { cuenta el contexto. }\end{array}$ & $\begin{array}{l}\text { La problemática está planteada, } \\
\text { sin embargo no está completa } \\
\text { ni presenta coherencia. }\end{array}$ & $\begin{array}{l}\text { La problemática no está } \\
\text { planteada en el texto. }\end{array}$ \\
\hline $\begin{array}{l}\text { Objetivo } \\
(0.5)\end{array}$ & $\begin{array}{l}\text { El propósito se logra y es evidente } \\
\text { a lo largo del texto. }\end{array}$ & $\begin{array}{l}\text { El propósito se mantiene en } \\
\text { general, aunque a veces se } \\
\text { pierde. }\end{array}$ & $\begin{array}{l}\text { El propósito no se evidencia } \\
\text { con claridad. }\end{array}$ \\
\hline $\begin{array}{l}\text { Enunciador y destinatario } \\
\qquad(0.5)\end{array}$ & $\begin{array}{l}\text { Nunca pierde de vista su rol y al } \\
\text { destinatario, mantiene el perfil } \\
\text { elegido desde la planificación. }\end{array}$ & $\begin{array}{l}\text { Pierde de vista al enunciador y } \\
\text { destinatario, pero regresa a él a } \\
\text { veces. }\end{array}$ & $\begin{array}{l}\text { No está dirigido al destinatario } \\
\text { correcto, ni el enunciador es el } \\
\text { adecuado. }\end{array}$ \\
\hline $\begin{array}{l}\text { Superestructura global } \\
\qquad(0.5)\end{array}$ & $\begin{array}{l}\text { El texto posee una estructura } \\
\text { canónicaqueincluye introducción, } \\
\text { desarrollo y conclusión de manera } \\
\text { articulada y cada una cumple su } \\
\text { función. Se evidencia claramente } \\
\text { la estructura problema-solución. } \\
\text { Se usan varias voces ajenas para } \\
\text { fundamentar las ideas. }\end{array}$ & $\begin{array}{l}\text { El texto contiene solo dos } \\
\text { partes. (Indique cuál parte de la } \\
\text { estructura global está ausente). } \\
\text { No se evidencia claramente la } \\
\text { estructura problema-solución. } \\
\text { Se usan pocas voces ajenas } \\
\text { para fundamentar las ideas. }\end{array}$ & $\begin{array}{l}\text { El texto posee una organización } \\
\text { débil y es difícil identificar sus } \\
\text { partes. } \\
\text { El texto no tiene la estructura } \\
\text { problema-solución. } \\
\text { No se usa ninguna voz ajena } \\
\text { en el texto. }\end{array}$ \\
\hline $\begin{array}{l}\text { Cohesión, coherencia y } \\
\text { claridad en la expresión de } \\
\text { las ideas (manejo de la } \\
\text { referencialidad) } \\
(0.75)\end{array}$ & $\begin{array}{l}\text { La redacción es clara y coherente, } \\
\text { manteniendo el sentido global de } \\
\text { la macroestructura. } \\
\text { Existe bastante cohesión entre las } \\
\text { ideas internas de cada párrafo, } \\
\text { presentándose oraciones tópicas } \\
\text { con sus explicaciones seguidas. } \\
\text { Se hace uso correcto de la } \\
\text { referencialidad en todo el texto. }\end{array}$ & $\begin{array}{l}\text { La redacción es generalmente } \\
\text { coherente, pero presenta } \\
\text { algunas ideas poco claras, } \\
\text { afectando el sentido global } \\
\text { de la macroestructura. Existe } \\
\text { poca cohesión entre las ideas } \\
\text { internas de cada párrafo, con } \\
\text { algunas oraciones tópicas y } \\
\text { sus explicaciones. Se hace uso } \\
\text { de la referencialidad solo en } \\
\text { algunas ocasiones en el texto. }\end{array}$ & $\begin{array}{l}\text { La redacción es poco clara } \\
\text { por la presencia de variadas } \\
\text { incoherencias y repeticiones. } \\
\text { Se pierde el sentido global } \\
\text { del texto. No existe cohesión } \\
\text { entre las ideas internas de } \\
\text { cada párrafo, ni oraciones } \\
\text { tópicas y sus explicaciones. } \\
\text { Uso nulo de la referencialidad } \\
\text { en el texto. }\end{array}$ \\
\hline $\begin{array}{l}\text { Normativa (respeto a } \\
\text { la normativa) } \\
(0.25)\end{array}$ & $\begin{array}{l}\text { Demuestra bastante dominio de la } \\
\text { nomativa. No presenta errores de } \\
\text { acentuación, uso de letras, puntuación } \\
\text { y concordancia. }\end{array}$ & $\begin{array}{l}\text { Aunque en el escrito se } \\
\text { respeta la normativa, presenta } \\
\text { algunos errores de acentuación, } \\
\text { uso de letras, puntuación y } \\
\text { concordancia. }\end{array}$ & $\begin{array}{l}\text { Presenta muchos errores de } \\
\text { acentuación, puntuación y } \\
\text { concordancia. }\end{array}$ \\
\hline $\begin{array}{l}\text { Soluciones propuestas } \\
\text { (1.5) }\end{array}$ & $\begin{array}{l}\text { Las soluciones propuestas } \\
\text { corresponden a cada uno de } \\
\text { los problemas planteados, } \\
\text { mostrando el pensamiento crítico, } \\
\text { reflexivo y analítico. }\end{array}$ & $\begin{array}{l}\text { Se plantean soluciones no } \\
\text { siempre de acuerdo con la } \\
\text { problemática, con algo de } \\
\text { criticidad. }\end{array}$ & $\begin{array}{l}\text { Las soluciones no están } \\
\text { relacionadas con la } \\
\text { problemática planteada en el } \\
\text { texto, son débiles, sin reflexión } \\
\text { ni criticidad. }\end{array}$ \\
\hline
\end{tabular}

«Implementación del proceso de producción escrita para el mejoramiento de la redacción de textos expositivos en Administración Hotelera», Kenia Rodríguez de Benítez. Cuaderno de Pedagogía Universitaria Año 12 / N.23 / enero-junio 2015 / Santiago, República Dominicana / PUCMM / p. 42-54 
Tabla 3

Cuestionario aplicado a estudiantes

\section{PONTIFICIA UNIVERSIDAD CATÓLICA MADRE Y MAESTRA \\ FACULTAD DE CIENCIAS SOCIALES Y ADMINISTRATIVAS \\ DEPARTAMENTO DE ADMINISTRACIÓN HOTELERA \\ ADH-335-T-002 Turismo Sostenible \\ Cuestionario evaluación estrategia del proceso de producción escrita}

Objetivo: Conocer la apreciación de los estudiantes sobre la estrategia de la escritura como proceso en la resolución del caso \#6 del Archipiélago de Cabrera.

I. Marque con una x su apreciación sobre el enunciado que se presenta a la izquierda.

\begin{tabular}{|c|c|c|c|c|}
\hline Criterio a evaluar & Muy de acuerdo & De acuerdo & $\begin{array}{l}\text { Medianamente de } \\
\text { acuerdo }\end{array}$ & En desacuerdo \\
\hline $\begin{array}{l}\text { Las instrucciones para aplicar } \\
\text { la estrategia fueron claras, } \\
\text { precisas y me orientaron bien } \\
\text { en el proceso. }\end{array}$ & & & & \\
\hline $\begin{array}{l}\text { Comprendí cómo aplicar la } \\
\text { estrategia desde el inicio. }\end{array}$ & & & & \\
\hline $\begin{array}{l}\text { El tiempo dedicado para } \\
\text { solucionar el caso fue el } \\
\text { adecuado. }\end{array}$ & & & & \\
\hline $\begin{array}{l}\text { El nivel de dificultad para aplicar } \\
\text { la estrategia fue el adecuado. }\end{array}$ & & & & \\
\hline $\begin{array}{l}\text { El paso no.1 (contextualizar) } \\
\text { le fue de mucha utilidad para } \\
\text { la generación de ideas para } \\
\text { redactar el texto. }\end{array}$ & & & & \\
\hline $\begin{array}{l}\text { El paso no. } 2 \text { (gestación de } \\
\text { ideas) le fue de mucha utilidad } \\
\text { para redactar el texto. }\end{array}$ & & & & \\
\hline $\begin{array}{l}\text { El paso no.3 (planificación del } \\
\text { texto) le fue de mucha utilidad } \\
\text { para redactar el texto. }\end{array}$ & & & & \\
\hline $\begin{array}{l}\text { La corrección del borrador } \\
\text { antes de la última entrega le } \\
\text { sirvió para mejorar el resultado } \\
\text { final del texto. }\end{array}$ & & & & \\
\hline $\begin{array}{l}\text { El uso de la rúbrica para evaluar } \\
\text { el texto fue de gran ayuda. }\end{array}$ & & & & \\
\hline $\begin{array}{l}\text { El uso de esta estrategia me } \\
\text { ayudó a reflexionar sobre las } \\
\text { mejores soluciones para el } \\
\text { caso. }\end{array}$ & & & & \\
\hline $\begin{array}{l}\text { Esta estrategia me servirá de } \\
\text { referencia para futuros trabajos. }\end{array}$ & & & & \\
\hline $\begin{array}{l}\text { Puedo decir que el uso de } \\
\text { esta estrategia para redactar } \\
\text { el texto, me convirtió en mejor } \\
\text { escritor(a). }\end{array}$ & & & & \\
\hline
\end{tabular}

"Implementación del proceso de producción escrita para el mejoramiento de la redacción de textos expositivos en Administración Hotelera», Kenia Rodríguez de Benítez. Cuaderno de Pedagogía Universitaria Año 12 / N.23 / enero-junio 2015 / Santiago, República Dominicana / PUCMM / p. 42-54 


\section{Presentación y análisis de los resultados}

Comparación de las evaluaciones obtenidas por los estudiantes del grupo experimental en el borrador y en el producto final, usando la rúbrica de evaluación.

En la fase del borrador, las principales observaciones realizadas a los estudiantes se enfocaron en: mejorar la cohesión y coherencia, definir con mayor claridad la problemática y mejorar la conclusión y soluciones propuestas. Las principales dificultades encontradas por los estudiantes estuvieron enfocadas en los criterios de coherencia, cohesión y claridad, así como la normativa.

En cuanto al producto final, las correcciones realizadas a los textos incluyeron elementos de la normativa, como acentuación, puntuación y concordancia. Los estudiantes mejoraron las conclusiones y las soluciones propuestas en el caso analizado. También, algunos errores de coherencia fueron cometidos, aunque en menor cantidad que en el borrador.

El borrador del caso analizado sacó a relucir la problemática descrita en este proyecto: la debilidad de los estudiantes para proponer soluciones a los problemas presentados en clase de una manera coherente y analítica. Además, se puede decir que la aplicación de la escritura como proceso, combinada con el uso de la corrección procesal, ayudó a mejorar los textos escritos, la coherencia y habilidad de los estudiantes para proponer soluciones.

En un sentido más global, se pudo evidenciar una mejora significativa entre la evaluación del borrador y del producto final, ya que se pasó de un $18 \%$ a un $57.1 \%$ de estudiantes que lograron en su mayoría los criterios en base a los cuales fueron evaluados, como muestra el gráfico 1.

\section{Gráfico 1}

Variación evaluaciones borrador y producto final del grupo experimental

\section{Variación Borrador y Producto Final}

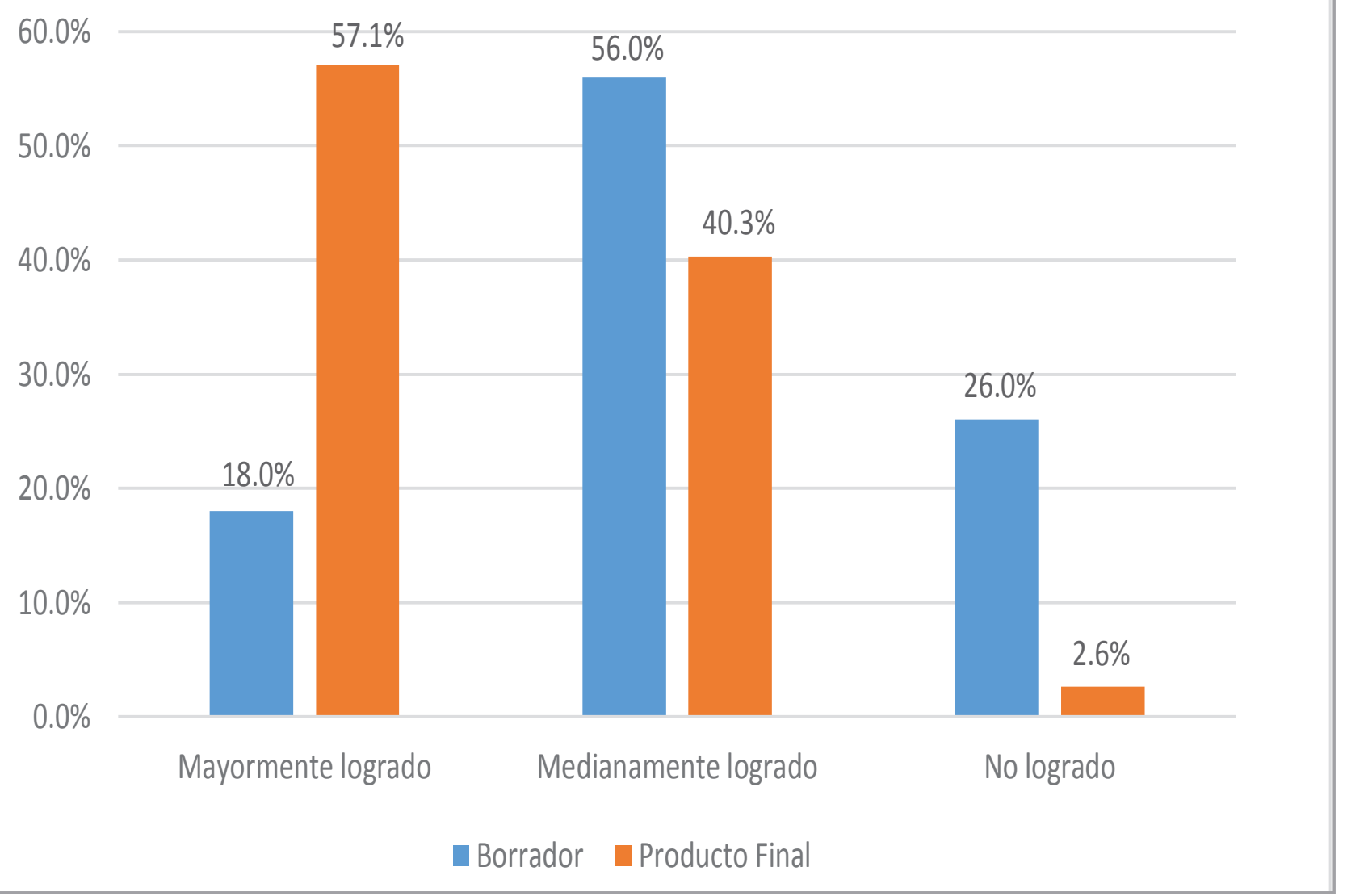

Comparación de las evaluaciones obtenidas en el caso asignado entre el grupo de control y el grupo experimental, usando la rúbrica de evaluación. 
La mayoría de los estudiantes del grupo control obtuvo calificaciones entre medianamente y no logrado. Las principales dificultades encontradas estuvieron relacionadas con la superestructura global del texto, ya que muchos no presentaron una estructura canónica compuesta por introducción, desarrollo y conclusión. En adición a esto, se evidenciaron grandes debilidades en la redacciónen cuanto a la coherencia, cohesión, claridad y normativa.
Al hacer una comparación entre las evaluaciones obtenidas por el grupo experimental y el grupo control, se observan grandes diferencias en los niveles alcanzados, ya que en el primero el $97.4 \%$ de estudiantes estuvo entre mayormente y medianamente logrado, mientras que en el segundo solo fue el $72 \%$ de los mismos, como se evidencia en el gráfico 2 .

\section{Gráfico 2}

Comparación evaluaciones grupo experimental y grupo control

\section{Evaluaciones Grupo Experimental y Grupo Control}

\section{$80.0 \%$}

$70.0 \%$ $67.0 \%$



A partir de estos datos se puede deducir que la aplicación de la estrategia de escritura como proceso puede ayudar a los estudiantes a obtener mejores resultados en sus producciones escritas, ofreciendo los pasos clave para redactar con mayor coherencia y reflexión cualquier texto expositivo. 
Resultados del cuestionario aplicado a los estudiantes para conocer su apreciación sobre la estrategia de la escritura como proceso.

En el cuestionario aplicado a los estudiantes para conocer su apreciación de la estrategia, se encontró que el $84 \%$ estuvo en la escala entre muy de acuerdo y de acuerdo con aspectos como: la claridad de las instrucciones, comprensión de la estrategia desde el inicio, tiempo y nivel de dificultad adecuados, la utilidad de los pasos del proceso de producción escrita, así como el uso de la rúbrica para revisar el texto.

Uno de los aspectos medidos fue la utilidad que los estudiantes le dieron a la retroalimentación recibida del borrador para mejorar el texto final, resultando en que el $72 \%$ estuvo de acuerdo con el beneficio de los comentarios recibidos. En otro orden, los estudiantes estuvieron de acuerdo en que el uso de esta estrategia les ayudó a reflexionar sobre las mejores soluciones para el caso y que les servirá para futuros trabajos.

Un elemento importante a destacar es que el $29 \%$ de los estudiantes estuvo muy de acuerdo en que el uso de esta estrategia para redactar el texto los convirtió en mejores escritores, el $50 \%$ de acuerdo, el $14 \%$ medianamente de acuerdo, mientras que el $7 \%$ estuvo de desacuerdo.

También se determinó que los pasos del proceso de la producción escrita que les parecieron más difíciles fueron los últimos tres: planificación del texto, redacción del borrador y el producto final, siendo la redacción del borrador el más difícil. Los primeros pasos, gestación de ideas y contextualización, los consideraron más fáciles de trabajar.

La mayoría de los comentarios de los estudiantes fueron positivos, expresando los beneficios de la estrategia y cómo les ayudó a ser más organizados y estructurados. También opinaron que aplicar el proceso de producción escrita les facilitó la búsqueda de soluciones y la reflexión. Sin embargo, se presentaron dificultades para algunos que no lograron comprender la estrategia porque implicaba muchas explicaciones y pasos. Otro elemento que salió a relucir fue el factor tiempo, ya que consideraron que ésta conlleva mucho esfuerzo y que en el aula se les complicaba expresar más libremente sus ideas.

Para futuras aplicaciones de la estrategia, éstos recomendaron expresar la guía con términos más comprensibles, mostrar más ejemplos a los estudiantes para aprender el estilo exigido, iniciar la práctica de manera progresiva y luego aumentar la dificultad, y que esta estrategia pueda ser aplicada en otro tipo de asignaciones para así lograr trabajos más estructurados y organizados.

\section{Conclusiones}

A través de los resultados encontrados en este proyecto, se evidencia que el uso de la estrategia de la escritura como proceso en la producción de textos expositivos con la estructura global problema-solución ayudó a mejorar en los estudiantes la capacidad para reflexionar y proponer soluciones de más calidad a las problemáticas presentadas en clase, como establece Cassany (1999, p. 16), "escribir es un poderoso instrumento de reflexión y que en el acto de escribir, los redactores aprenden sobre sí mismos, sobre su mundo y comunican sus percepciones a otros".

Sobre la implementación del proceso de producción escrita en todas sus etapas fue gratificante ver cómo los estudiantes lograron gestar mejor sus ideas, planificar su texto con las instrucciones dadas y redactar los borradores, para luego concluir con un producto final con mejoras significativas, siguiendo un proceso cognitivo como es descrito por Flower y Hayes (1981).

Está claro que los docentes no deben esperar que los estudiantes dominen todas las áreas de la composición escrita, sino que su principal papel es el de guía y colaborador con el alumno a través de una corrección procesal cuyo objetivo no es calificar los trabajos como buenos y malos, sino retroalimentar con miras hacia las mejoras. Definitivamente, la corrección procesal es una herramienta válida para lograr avances en los estudiantes y "debe ser un instrumento eficaz para el aprendizaje si se sabe utilizar con inteligencia" (Cassany, 2002, p. 28).

En cuanto a la apreciación de los estudiantes de la estrategia aplicada, se manifestó que la mayoría de éstos estuvo de acuerdo con los beneficios que les aportó la misma. Estos valoraron como positivo haber recibido retroalimentaciones a través del borrador y la mitad de ellos expresó estar de acuerdo en que esta estrategia los convirtió en mejores escritores. Sin embargo, es importante resaltar que estos demostraron tener mayor facilidad para pasos sencillos como la gestación de ideas y contextualización, presentado retos a la hora de pasar a procesos más elaborados y complejos. En este sentido, es el deber del docente ser facilitador aplicando estrategias que los lleven hacia un mayor grado de elaboración, como es el caso de este estudio, con textos con la estructura problema/solución. 
Algunos retos presentados en el proyecto estuvieron relacionados con el factor tiempo, ya que las correcciones debían ser lo más específicas posibles, implicando mayor dedicación y minuciosidad en las retroalimentaciones. Además, una minoría de los estudiantes no logró comprender la estrategia ni lo que se pretendía con ésta, lo que podría sugerir que, al incluir tantos pasos e instrucciones, en algunos casos podría generarse confusión. Se podría iniciar, como proponen los estudiantes en las recomendaciones, por niveles más bajos de exigencia del proceso e ir aumentando la dificultad según los avances de los mismos.

Definitivamente, la escritura representa grandes retos para el estudiantado y está en las manos del docente colaborar con estrategias que ayuden en este sentido. Escribir no es tan sencillo como parece, no se puede esperar que los estudiantes sean expertos en esta área. Es el deber de la comunidad académica la aplicación de estrategias que vayan encaminando hacia el aprendizaje significativo.

\section{Recomendaciones}

Para futuros proyectos de investigación-acción relacionados con la estrategia de la escritura como proceso a través de textos expositivos problemasolución, sería importante tomar en cuenta las siguientes recomendaciones:

- Reforzar en los estudiantes las habilidades de escritura, partiendo de la premisa de que éstos necesitan apoyo, ofreciendo espacios dentro de las asignaturas diseñados con criterio e implementando la corrección procesal.

- Iniciar la aplicación de la estrategia con tiempo suficiente para poder realizar una retroalimentación con mayor profundidad y guía.

- Explicar más detalladamente las instrucciones del proceso de producción escrita, para evitar confusiones en algunos estudiantes que requieren de un mayor seguimiento. Si es posible, mostrar mayor cantidad de ejemplos de textos expositivos problema-solución con anticipación para familiarizar a los estudiantes.

- Solicitar a todos los estudiantes que registren sistemáticamente los pasos que siguieron para la redacción y entregarlos junto con el borrador. De esta forma, se puede ayudar al estudiante a ser consciente de su propio proceso, es decir, lograr la metacognición.

\section{Referencias}

Arias, S. (2013). Mejoramiento de la calidad de las producciones escritas de estudiantes de Administración de Empresa en sus informes de Pasantía Empresarial. Diplomado en lectura y escritura a través del currículo en el nivel superior. Pontificia Universidad Católica Madre y Maestra.

Carlino, P. (2013). Alfabetización Académica Diez Años Después. Revista Mexicana de Investigación Educativa, vol. 18, núm. 57, pp. 355-381. Consejo Mexicano de Investigación. Recuperado de: http:// www.redalyc.org/articulo.oa?id=14025774003

Cassany, D. (2002). Reparar la Escritura. Didáctica de la corrección de lo escrito. Barcelona: Editorial Graó.

Cassany, D., Luna, M. \& Sanz, G. (2002). Enseñar lengua. Barcelona: Editorial Graó.

Cassany, D. (1999). Construir la Escritura. Barcelona: Ediciones Paidós Ibérica.

Castelló, M., Iñesta, A. \& Monereo, C. (2009). Hacia la escritura académica autorregulada: un estudio exploratorio con estudiantes posgraduados en un entorno de aprendizaje situado. Electronic Journal of Research in Educational Psychology, 7(3).b Recuperado de: http://www.investigacionpsicopedagogica.com/revista/new/index.php?n=19

Flower, L. \& Hayes, J. R. (1981). A cognitive process theory of writing. College Composition and Communication, 32(4), 365-387. National Council of Teachers of English. Recuperado de:http://kdevries. net/teaching/teaching/wp-content/uploads/2009/01/ flower-hayes-81.pdf

Huerta, J. (2012). Escritura por proceso: la planeación de lo escrito. Memoria de Ponencias. III Seminario Internacional de Lectura en la Universidad. II Congreso Nacional de Expresiones de Cultura Escrita en Instituciones de Educación Media Superior y Superior. IV Seminario Internacional de Cultura Escrita y Actores Sociales. México.

Padilla, C., Douglas, S. \& López, E. (2010). Yo Expongo: Taller de prácticas de comprensión y producción de textos expositivos. Córdoba: Comunicarte Editorial.

Plan de Estudios de la Carrera Administración Hotelera. (2012). Programa Académico Asignatura ADH-335 Turismo Sostenible. Pontificia Universidad Católica Madre y Maestra. 